\title{
Proliposomes as Pharmaceutical Drug Delivery System: A Brief Review
}

\author{
Muneer $\mathbf{S}^{1,2,3 *}$, Masood $\mathbf{Z}^{1}$, Butt $\mathbf{S}^{1}$, Anjum $\mathbf{S}^{1}$, Zainab $\mathbf{H}^{1}$, Anwar $\mathbf{N}^{1}$ and Ahmad $\mathbf{N}^{1}$ \\ ${ }^{1}$ Faculty of Pharmacy, University of Lahore, Pakistan \\ ${ }^{2}$ Faculty of Pharmacy, University of Karachi, Pakistan \\ ${ }^{3}$ Pharmacy Australia Center of Excellence, University of Queensland, Australia
}

\begin{abstract}
Liposomes, the microscopic vesicles, are composed of a bilayer of phospholipids and a novel drug delivery system that provides drug at predetermined rate decided according to need, pharmacological aspects, drug profile, physiological conditions of body etc. But liposomes exhibit poor stability problem and this leads to their storage problem. To circumvent this problem Pro-liposomes (PLs) were discovered in 1986. Pro-liposomes are free flowing granular products composed of drug and phospholipid precursors which on hydration lead to liposomes. This paper reviews various features regarding pro-liposomes, their method of preparation, evaluation, applications and pointing out its potential to be employed for different routes of administration.
\end{abstract}

Keywords: Liposome; Pro-liposome; Carriers; Phospholipids; Cholesterol

\section{Introduction}

The liposomes were first described by British haematologist Dr. Alec D Bangham in 1961 at the Babraham Institute, in Cambridge. Liposome is derived from Greek words: "Lipos" meaning fat and "Soma" meaning body [1]. Out of all the novel drug delivery systems, liposomes are considered to be the most effective, researched and broadly [2]. A micro spherical vesicle consisting of an aqueous core enclosed in phospholipid molecules is known as liposome. Drug molecules can be incorporated into the aqueous phase or within the lipid bilayer. They are widely used as a vehicle for administration of nutrients and pharmaceutical drugs to improve the stability and effectiveness of drug by reducing the side effects [3]. To enter the market, Liposomes should remain stable and intact during the storage period and before reaching the targeted site to produce therapeutic action. However, due to physical and chemical instability, liposomes are relatively unstable colloidal systems [4].

Liposomal suspension may have limited shelf life and to overcome the stability issue associated with liposome, a new "pro-liposome" method is developed that can produce liposomes quickly when there is a need and without excessive manipulation [5]. Pro-liposomes (PLs) were discovered in 1986 [6]. Pro-liposomes are dry, free-flowing granular products that on hydration or on contact with biological fluids in the body, form liposomal dispersion. They are composed of water soluble porous powder and phospholipid [7].

For producing commercial liposome products, Pro-liposome is one of the most widely used and cost-effective methods. As they are available in dry powder form, it's easy to distribute, transfer, measure and store, making it a diverse system. Liposomes can either be formed in vivo by the influence of biological fluids in the body or in vitro using a suitable hydrating fluid before the administration [8]. Solubility and bioavailability problems of many drugs can be overcome by developing pro-liposomal formulations [9].

\section{Method of Preparation}

Pro-liposomes (PLs) are prepared by many methods such as:

- Film-deposition on carrier method.

- Spray drying method.
- Fluidized-bed method.

- Supercritical anti-solvent method.

\section{Film deposition on carrier method}

Film deposition on carrier method is used for the composition of Pro-liposomes. In this procedure, the coat of drug and phospholipids is discharged on a pervious, water soluble carrier substance. By viewing Figure 1, an evaporative solution containing a solution of drug and phospholipids is injected drop by drop by a feed tube onto a core of carrier substance which is carried in a vessel of a rotary flash evaporator under vacuum. At any stated moment, the matrix's overwetting is circumvented and following aliquot of organic mixture is feeding solely when a free flowing powder matrix is procured [10]. Selected carriers should exhibit great surface area and permeability in order to regulate the quantity of carrier which is needed to assist the lipids. This also permits great surfactant to carrier mass proportion for the pro-liposomes production. As they are water soluble, they enable fast production of liposomal dispersion on hydration and by properly managing the size of pervious powder, comparatively limited variety of reconstituted liposomes can be acquired. Mostly used carriers are maltodextrin, sorbitol, microcrystalline cellulose, magnesium aluminum silicates, mannitol, etc. [11]. Stride of solvent inclusion and evaporation which is sluggish [12]. To circumvent this issue, alter the procedure by dispersing the carrier substance in organic mixture of drug and phospholipids in vessel of rotary evaporator and then directing it to vacuum evaporation. By doing so, highly consistent and well-organized lipid distribution is achieved and a steady and less time taking procedure is gained in contrast to the actual procedure [13].

\section{Spray drying method}

The distinctive attribute of this process is reclined in its propensity

*Corresponding author: Saiqua Muneer, University of Queensland, Australia, Tel: 61469085227; E-mail: muneer@uq.edu.au

Received: June 07, 2017; Accepted: June 24, 2017; Published: June 29, 2017

Citation: Muneer S, Masood Z, Butt S, Anjum S, Zainab H, et al. (2017) Proliposomes as Pharmaceutical Drug Delivery System: A Brief Review. J Nanomed Nanotechnol 8: 448. doi: 10.4172/2157-7439.1000448

Copyright: @ 2017 Muneer S, et al. This is an open-access article distributed under the terms of the Creative Commons Attribution License, which permits unrestricted use, distribution, and reproduction in any medium, provided the original author and source are credited. 


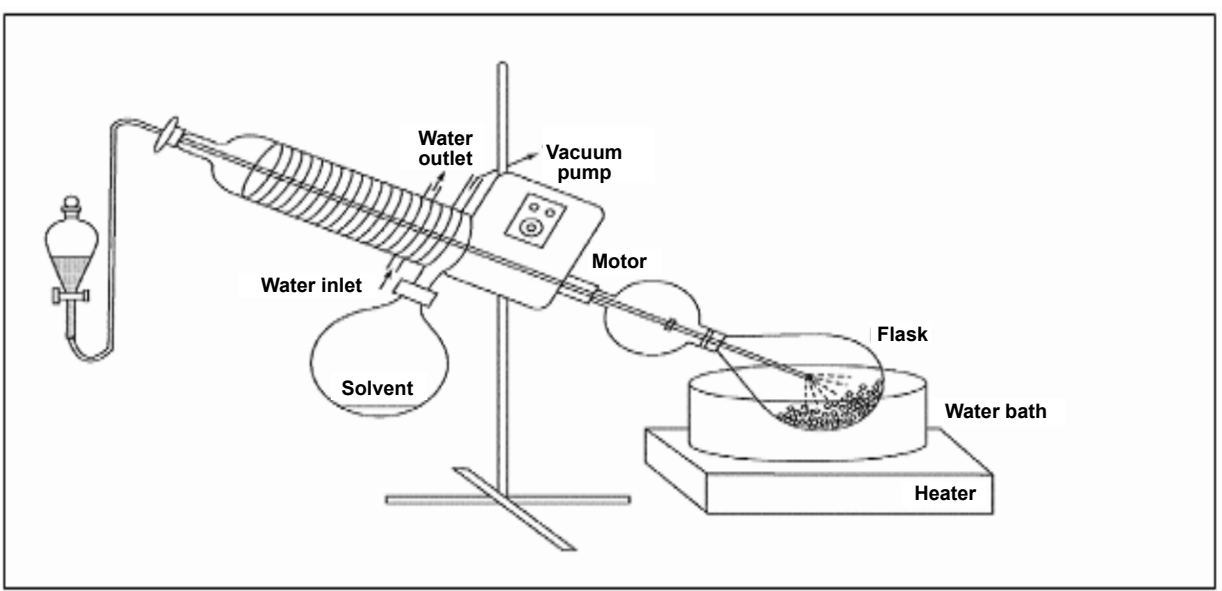

Figure 1: Apparatus for preparing PLs by film deposition on Carrier method.

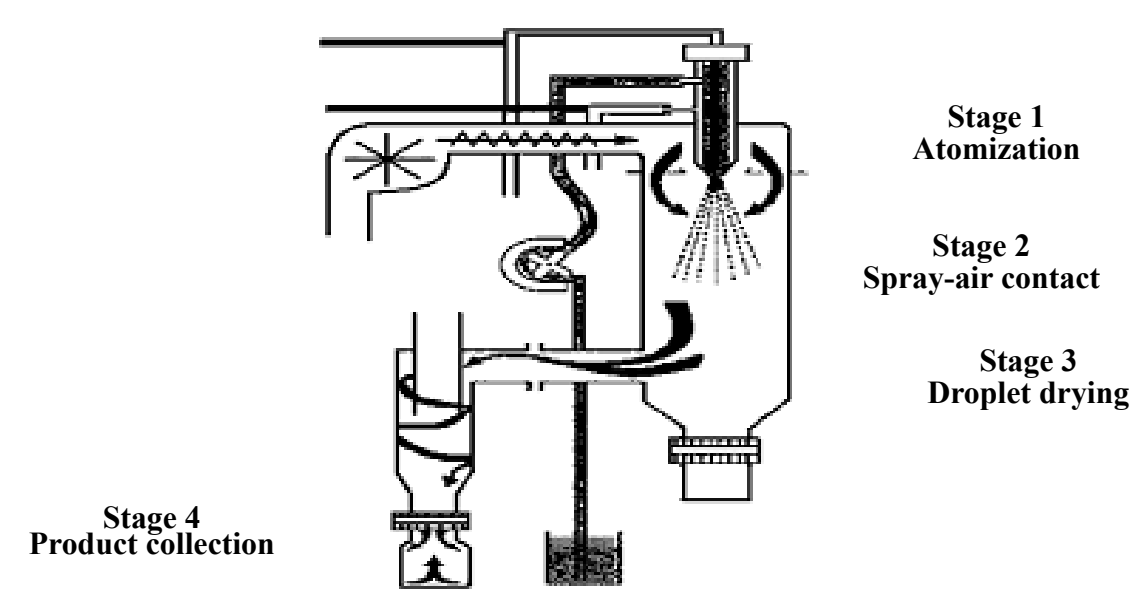

Figure 2: Apparatus for preparing PLs by Spray drying method.

to include particle composition and drying together in a consistent stride, permitting more desirable production of particles. This method can be used for any of the aqueous or non-aqueous systems for particles production. Predominantly, this process is utilized when invariable sized and shaped particles are needed and can be simply scaled up. Its price is effectual and acceptable for massive preparation of PLs $[14,15]$.

As shown in Figure 2, this spray drying procedure consists of four phases: atomization of the product into a spray nozzle, spray-air association, drying of the spray droplets and collection of the solid product [16]. Firstly, preparation of liquid dispersions carrying pure lipid or lipids and carriers in organic mixture is done and then it is poured into the dry cell. By utilizing a spray nozzle, dispersions are atomized into drying cell and desiccated in a simultaneous air flow which is then gathered in a tank [16]. Prime factors which affecting this method are high temperatures, shearing stresses and absorption episodes and these can result into the thermal and mechanical degradation of active molecules. It can be upgraded by making the working variables better. Examples of working variables are drying air temperatures and liquid spraying rate. For shielding the unification of active molecules, stabilizing adjuvants e.g. disaccharides, cyclic oligosaccharides and polyols can be utilized and by augmenting the surface area of lipids, the effectiveness of hydration can be intensifying $[14,15]$.

\section{Supercritical anti-solvent method}

In Super critical anti-solvent method for the production of PLs we use Supercritical Carbon dioxide $\left(\mathrm{SCCO}_{2}\right)$ which actually is carbon dioxide's fluid state when it is held at on some level above its critical temp and pressure.

Because of three main factors which includes

- Lower residual solvents,

- Simple steps,

- Mild operation temperatures.

We use anti-solvent technology for the preparation of PLs. An apparatus consisting of three parts (e.g. a sample delivery unit, a precipitation unit and a separation unit) is basically used in those simple steps. Two pumps, one for the delivery of $\mathrm{CO}_{2}$ which is supplied through $\mathrm{CO}_{2}$ cylinder $\left(72 \mathrm{~cm}^{3}\right)$ after being cooled down by refrigerator and a high pressure pump is used introduce it to the buffer tank $\left(-7^{\circ} \mathrm{C}\right)$ for preheating thus The conditions of temperature and pressure of the 
reaction vessel or $\mathrm{CO}_{2}$ cylinder should be $45^{\circ} \mathrm{C}$ and $10 \mathrm{MPa}$ and one for the drug solutions which is introduced via HPLC pump combines up to make the sample delivery unit [17]. Solvent which are completely miscible with $\mathrm{CO}_{2}$ should be used for dissolving the drugs. For both preparations, phospholipids, cholesterol, and drug were dissolved in organic solvents followed by sonication until a clear and homogeneous solution was obtained. For the entrance of $\mathrm{CO}_{2}$ into the vessel through nozzle valves $\mathrm{A}$ and $\mathrm{B}$ will be opened. $\mathrm{CO}_{2}$ is sprayed through the outer tubule whereas the solution is sprayed though inner tubule of the nozzle. The second part of the apparatus consists of heated by air bath vessel, and the last part comprises wet gas meter and a separator. $\mathrm{SCCO}_{2}$ is separated from organic solvent in the last part's separator because of its low pressure and on the other hand wet gas meter is used to measure the $\mathrm{CO}_{2}$ [18]. After reaching the preset value of temp and pressure, valve $\mathrm{A}$ is open for the entrance of $\mathrm{CO}_{2}$ right after that, valve $\mathrm{B}$ allow drug solution to enter the nozzle. Solution is mixed with $\mathrm{SCCO}_{2}$ and diffused into each other rapidly like it is sprayed through coxial nozzle. Thus the solute will dissolve in organic solvent to reach supersaturation in a very short period of time about 30 minutes and this all because of the solubility of solute in the organic solvent decreases gently, thus the PLs are precipitated in the vessel. After the complete utilization of solution, $A$ and $B$ valves are closed and valve $C$ is opened to depressurize the vessel at the opening temp and in the end we collect these samples at the bottom of vessel on the filter. The pressure, temp and the flow rate of the drug solution need to be optimized to obtain the high drug loading PLs.

\section{Fluidized bed method}

On the large scale production of PLs whose principle relays on particle coating technology, in which carrier material can vary from crystalline powder to non pareil beads. While using non pareil beads as carrier material, first for getting smooth surface pareil beads are coated with seal coating which can help further in coating of phospholipids and which also ensure thin uniform coating formation of phospholipids around the core and small sized liposomes upon hydration. Carrier material are then sprayed with the solution of organic solvent and solution of drugs through nozzle, and by applying vacuum at the same time to the fluid bed organic solvent is removed. The trace amount of residual solvent is removed by the finished lipid-coated powder/beads when dried under vacuum overnight (Figure 3).

Advantages include;

- Well establish and processable Film coating technology
- Number of various ways of coating

- Cost effective method [19,20].

\section{Evaluation of Proliposome}

\section{Scanning electron microscopy (SEM)}

It is used to observe surface structure of the PL powder. It includes the comparison of the image of liposome and pure carrier material. Carrier material in the formulation confirms the disposition of phospholipids on the carrier and thus proliposomes formulation confirms [21]

\section{Transmission electron microscopy (TEM)}

This method is also used to check the structure of liposomes after PL powder hydration. In this process hydrate the proliposome's powder with distilled water and then view lamellarity and the shapes under microscope $[8,10,21]$.

\section{Hydration study}

Hydration study is carried out on the fact that liposomes are formed on contact with aqueous environment. In this method we place small quantity of dry powder of pro-liposomes and place it on a glass slide and then gradual addition of water in it and is observed by using microscope to view vesicle formation. During hydration dissolution and disintegration occur rapidly as soon as hydration. Liposomes are formed when the water come in contact with the lipid surface of proliposomes. This process continuous tills the complete hydration lipid layer and carrier dissolution [22].

\section{Zeta potential}

Surface charge of particle can be determined by the zeta potential. It is the potential difference between electro neutral region of the solution and surface of tightly bound layer (shear place) [23].

\section{Flow property}

Content uniformity and handling processing operation can be explained by the flow property of a powder formulation. For a formulation based on solid powder it is necessary to analyse the pro-liposome's property. It can be assessed by measuring following parameters; Angle of repose, Carr's Index and Hausner's ratio [11,22].

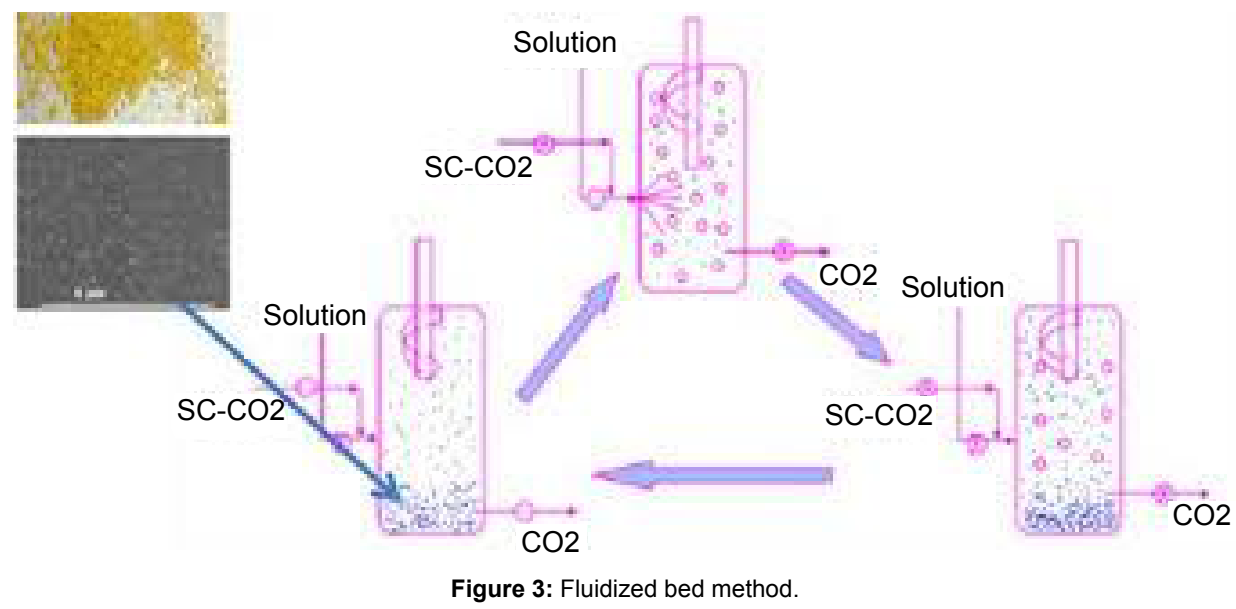




\section{Applications}

Pro-liposomes can be formulated for the below mentioned routes of administration.

\section{Parenteral delivery}

For parenteral application, most important is their sterilization. Sterilization techniques commonly used are steam sterilization, $\gamma$-irradiation, aseptic manufacturing and filtration sterilization. Terminal sterilization is not appropriate for liposomal formulations as it requires steam at $121^{\circ} \mathrm{C}$. At high temperature liposome structure is destroyed because of lipid hydrolysis and boosts the per oxidation of unsaturated lipids [24,25]. Pro-liposomes are adequate for parenteral delivery of liposomes. The benefit linked with Pro-liposomes is that it permits sterilization without affecting the intrinsic characteristics [26]. Pro-liposomes can be stored in dry form after sterilization and can be hydrated before administration to form multi-lamellar liposomal suspension [27]. In the past few decades, Pro-liposomes played a vital role in the area of injectable drug delivery system. Entrapment of drug into multi-vesicular liposomes leads to novel approach in sustained release drug delivery. Liposomal entrapment of drug results in sustained release lasting over several days to weeks [28].

\section{Oral delivery}

Pro-liposomes help to enhance the dissolution efficiency of poorly soluble drugs. It produces multi-lamellar vesicles on contact with fluid which guarantees higher entrapment of insoluble drugs due to widened hydrophobic volume within the liposomal lamellae. It also permits conversion of drug from crystalline to amorphous form [2]. Improvement in bioavailability of drugs having extensive first pass metabolism and increased lymphatic uptake is due to larger particle size of multi-lamellar liposomes formed on hydration [29]. Pro-liposomes are formulated to increase stability of liposomes. Formulations improve solubility and bioavailability of some poorly soluble drugs. Domperidone is a specific 5HT3 receptor antagonist used as anti-emetic. Domperidone is poorly soluble in water and after oral administration it undergoes extensive gastric and hepatic first pass metabolism. This leads to very low oral bioavailability that will not produce its required therapeutic effect. Pro-liposomes of Domperidone are formulated in order to increase bioavailability by enhancing intestinal permeability that results in improved lymphatic uptake and circumventing first pass metabolism [30]. Pro-liposomal formulations are now extensively used for drugs having low aqueous solubility like Exemestane [31], Salamon Calcitonin [32], Glyburide [33], Halofantrine [34] and Progesterone [35].

\section{Pulmonary delivery}

Liposomal preparations are also formulated for localized drug action in the respiratory tract. As liposomes are composed of phospholipids that are also a part of lung surfactant thus, drug entrapment within the liposomes result in improved absorption. Drugs encapsulated in Liposome are present in blood for extended period of time and with decreased adverse effects [36,37]. Pulmonary drug delivery is attained by following three types of devices:

Pressurized metered dose inhalers (pMDI): Drugs solution or suspension are added to liquefied propellants. Hydrofluroalkanes are used instead of Chlorofluorocarbons as they are non-ozone depleting propellants but they are poorly soluble in phospholipids. Pro-liposomes can be suspended in these propellants and act as as carrier of liposomes for pulmonary route [36].
Dry powder inhalers (DPIs): It involves the inhalation of drug as fine powder that causes dispersion of drug directly into airstream of patient. Dry powder inhalers have numerous benefits like controlled delivery, decreased toxicity and enhanced potency, even displacement of drugs locally, increased patient compliance, improved stability and large amount of drug entrapment. Pro-liposome formulations are in dry powder form so they are used preferably for liposomes delivery by dry powder inhaler [36]. Spray dried liposomes entrapped Dapsone dry powder inhaler have extended drug action in lungs to impede Pneumocystis carinii pneumonia. In vitro studies predict 16 hours prolonged drug release [38].

Nebulizers: It is the simplest method for providing liposomes to the respiratory system but it can be upset by liposome leakage and drug instability. Dry powder formulations are used to get rid of this problem. So, pro-liposomes are strong carriers of liposomes through nebulization [36,37].

Mucosal delivery: Pro-liposomes on contact with aqueous mucosal surfaces transformed into liposomes. Phospholipids are the component of pro-liposomes which are non-toxic, non-irritant and compatible with biological membranes. The molecular dispersion of drug into bilayer results in increased therapeutic action [39]. The vaginal proliposomal formulation of Clotrimazole has prolonged release of drug and may improve the drug retention time within the mucosa that leads to better antifungal effect [40].

Transdermal delivery: Pro-liposomes are composed of phospholipids that have natural affinity for skin lipids and thus enhance the drug permeation within the skin. Pro-liposomes on hydration are converted to liposomes that lead to sustained action of entrapped drug. Liposomes developed on contact with aqueous environment regulate diffusion across the skin. Hence the permeation of skin is increased by avoiding the stratum corneum hindrance [40,41]. Aceclofenac and Nicotine proliposomal formulations have been formulated for sustained transdermal action [41].

Ocular drug delivery: Conventional ocular drugs have poor bioavailability because of precorneal loss effects. Pro-liposomes are used to enhance the drugs bioavailability and their therapeutic action. Drugs entrapped within the lipid bilayer of liposomes have high solubility and can traverse cornea. Liposomal formulations can easily be used for ocular drug delivery. Liposomal hydrogels of Ciprofloxacin are used in order to prevent catheters from bacteria [41].

\section{Conclusion}

Pro-liposomes have provided a major breakthrough in solving the stability issues associated with liposomes. It has also opened newer areas of liposome application. Significant progress has been made towards the development of pro-liposomes as useful oral dosage forms although there are no marketable products. Reviews on oral pro-liposomes have highlighted several issues of importance that include complexity of experimental design, variability in response ,multiplicity of formulation variables and stability problems in GI tract. Pro-liposome have been used on a broad range of pharmaceutical application. The pro-liposomes are administrated orally, parenterally and topically as well as used in cosmetic and hair technologies, sustained release formulations, diagnostic purpose and as good carriers in gene delivery. Nowadays pro-liposomes are used as versatile carriers for targeted delivery of drug. However based on these we can say that Pro-liposome have definitely established their position in modern delivery system. 
Citation: Muneer S, Masood Z, Butt S, Anjum S, Zainab H, et al. (2017) Proliposomes as Pharmaceutical Drug Delivery System: A Brief Review. J Nanomed Nanotechnol 8: 448. doi: 10.4172/2157-7439.1000448

\section{References}

1. Dua JS, Rana AC, Bhandari AK (2012) Liposome: methods of preparation and applications. Int J Pharm Stud Res 3: 14-20.

2. Hiremath PS, Soppimath KS, Betageri GV (2009) Proliposomes of exemestane for improved oral delivery: formulation and in vitro evaluation using PAMPA, Caco-2 and rat intestine. Int J pharm 380: 96-104.

3. Sun C, Wang J, Liu J, Qiu L, Zhang W, et al. (2013) Liquid proliposomes of nimodipine drug delivery system: preparation, characterization, and pharmacokinetics. Aaps Phar 14: 332-338.

4. Yadav A, Murthy MS, Shete AS, Sakhare S (2011) Stability aspects of liposomes. Ind J Pha Edu 45: 402-413.

5. Uchegbu LF (2000) Publisher: CRC Press, 93-96.

6. Payne NI, Timmins P, Ambrose CV, Ward MD, Ridgway F (1986) Proliposomes: a novel solution to an old problem. J pha sc 75 : 325-329.

7. Manjula D, Shabaraya AR, Shyale S (2014) Topical Delivery of Fenoprofen Proliposomes: Preparation, Evaluation and In Vitro Release. Int J Pha Sc In 3: 06-12.

8. Janga KY, Jukanti R, Velpula A, Sunkavalli S, Bandari S, et al. (2012) Bioavailability enhancement of zaleplon via proliposomes: Role of surface charge. Eur J Phar Bio 80: 347-357

9. Ashok K, Jukanti R (2012) Euro J Phar Bio, pp: 347-357.

10. Song KH, Chung SJ, Shim CK (2002) Preparation and evaluation of proliposomes containing salmon calcitonin. J Con Rel 84: 27-37.

11. Janga KY, Jukanti R, Velpula A, Sunkavalli S, Bandari S, et al. (2012) Bioavailability enhancement of zaleplon via proliposomes: Role of surface charge. Euro J Phar Biop 80: 347-357.

12. Rojanarat W, Changsan N, Tawithong E, Pinsuwan S, Chan HK, et al. (2011) Isoniazid proliposome powders for inhalation-preparation, characterization and cell culture studies. Intern J Mol Sci 12: 4414-4434.

13. Xu H, He L, Nie S, Guan J, Zhang X, et al. (2009) Optimized preparation of vinpocetine proliposomes by a novel method and in vivo evaluation of its pharmacokinetics in New Zealand rabbits. J Con Rel 140: 61-68.

14. Lo YL, Tsai JC, Kuo JH (2004) Liposomes and disaccharides as carriers in spray-dried powder formulations of superoxide dismutase. J Con Rel 94: 259-272.

15. Colonna C, Conti B, Genta I, Alpar OH (2008) Non-viral dried powders for respiratory gene delivery prepared by cationic and chitosan loaded liposomes. Inter J Phar 364: 108-118.

16. Alves GP, Santana MHA (2004) Phospholipid dry powders produced by spray drying processing: structural, thermodynamic and physical properties. Pow Tech 145: 139-148.

17. Vyas SP, Khar RK (2002) Submicron emulsions in targeted and controlled drug delivery. Novel Carrier Systems.

18. Alves GP, Santana MHA (2004) Phospholipid dry powders produced by spray drying processing: structural, thermodynamic and physical properties. Pow Techn 145: 139-148.

19. Chen CM, Alli D (1987) Use of fluidized bed in proliposome manufacturing. $J$ Phar Sci 76: 419-419.

20. Liu R (2008) Water-insoluble drug formulation. CRC Press.

21. Yan-Yu X, Yun Mei S, Zhi-Peng C, Qi-Neng P (2006) Preparation of silymarin proliposome: a new way to increase oral bioavailability of silymarin in beagle dogs. Inter Phar 319: 162-168.

22. Payne NI, Browning I, Hynes CA (1986) Characterization of proliposomes. J Phar Sciences 75: 330-333

23. Martin A (1993) Physical pharmacy: physical chemical principles in the pharmaceutical sciences: BI Waverly Pvt. Ltd.

24. Burgess DJ (2005) Injectable dispersed systems: Formulation, processing, and performance. Boca Raton: Taylor \& Francis.
25. Vemuri S, Rhodes C (1995) Preparation and characterization of liposomes as therapeutic delivery systems: a review. Pharm Ac Hel 70: 95-111.

26. Song KH, Chung SJ, Shim CK (2002) Preparation and evaluation of proliposomes containing salmon calcitonin. Journal of Controlled Release 84: 27-37.

27. Hwang BY, Jung BH, Chung SJ, Lee MH, Shim CK (1997) In vitro skin permeation of nicotine from proliposomes. Journal of Controlled Release 49 177-184.

28. Bari $\mathrm{H}$ (2010) A prolonged release parenteral drug delivery system-an overview. Int J Pharm Sci Rev Res 3: 1-11.

29. Kumar R, Gupta RB, Betegeri G (2001) Formulation, characterization, and in vitro release of glyburide from pro-liposomal beads. Drug Delivery 8: 25-27.

30. Nalla P, Bagam S, Eedara BB, Dhurke R (2015) Formulation and Evaluation of Domperidone Oral Proliposomal Powders. International Journal of Pharmatech Research 7: 108-118.

31. Song KH, Chung SJ, Shim CK (2002) Preparation and evaluation of proliposomes containing salmon calcitonin. Journal of controlled release 84 $27-37$

32. Kumar R, Gupta RB, Betegeri G (2001) Formulation, characterization, and in vitro release of glyburide from proliposomal beads. Drug Delivery 8: 25-27.

33. Brocks DR, Betageri GV (2002) Enhanced oral absorption of halofantrine enantiomers after encapsulation in a proliposomal formulation. Journal of Pharmacy and Pharmacology 54: 1049-1053.

34. Chen Y, Lu Y, Chen J, Lai J, Sun J, et al. (2009) Enhanced bioavailability of the poorly water-soluble drug fenofibrate by using liposomes containing a bile salt. International Journal of Pharmaceutics 376: 153-160.

35. Taylor KM, Elhissi AM (2006) Preparation of liposomes for pulmonary delivery using medical nebulizers. Liposome Technology Liposome Preparation and Related Techniques 1: 67-84.

36. Sweeney LG, Wang Z, Loebenberg R, Wong JP, Lange CF, et al. (2005) Spray freeze dried liposomal ciprofloxacin powder for inhaled aerosol drug delivery. International Journal of Pharmaceutics 305: 180-185.

37. Chougule M, Padhi B, Misra A (2008) Development of spray dried liposomal dry powder inhaler of dapsone. Aaps Pharmscitech 9: 47-53.

38. Arora P, Sharma S, Garg S (2002) Permeability issues in nasal drug delivery Drug Discovery Today 7: 967-975.

39. Hwang BY, Jung BH, Chung SJ, Lee MH, Shim CK (1997) In vitro skin permeation of nicotine from proliposomes. Journal of Controlled Release 49 177-184.

40. Jukanti R, Sheela S, Bandari S, Veerareddy PR (2011) Enhanced bioavailability of exemestane via proliposomes based transdermal delivery. Journal of Pharmaceutical Sciences 100: 3208-3222.

41. Hiremath R, Gowda D, Raj A, Shamant BS, SrivastavaA, et al. (2016) Proliposomes: A novel approach to carrier drug delivery system. Journal of Chemical and Pharmaceutical Research 8: 348-354. 